Acta Crystallographica Section A

Foundations of

Crystallography

ISSN 0108-7673

\section{Supplementary material to: On the effect of neglecting anharmonic motion in charge density studies}

\author{
Kathrin Meindl, Regine Herbst-Irmer and Julian Henn * \\ ${ }^{a}$ Georg-August Universität Göttingen, Tammannstrasse 4, 37077 Göttingen Germany. Correspondence \\ e-mail: jhenn@chemie.uni-goettingen.de
}

\section{Supplementary material}

The following Table gives the details of the density- and thermal motion parameters of the $\mathrm{P}$ atom after multipole refinement.
The same data was used for the graphical representations in the main paper. Estimated standard uncertainties (s.u.) are also given.

Table 1

Selected geometry-, density-, and thermal motion parameters of the $\mathrm{P}$ atom in $\mathbf{1}$ in the noise-free, ideal reference model (= true parameters) (a), after refinement excluding noise and anharmonic motion in the refinement (b), after introduction of noise including full anharmonic motion (c), after refinement neglecting anharmonic motion at the $\mathrm{Al}$ atom (d), after refinement excluding anharmonic motion of $4^{\text {th }}$ order at the $\mathrm{P}$ atom (d) and finally excluding anharmonic motion at all (f) but including experimental noise. Distances are given in $\AA$, Uij in $\AA^{2}$, Cijk in $\AA^{3}$, Dijkl in $\AA^{4}$, monopoles in e, $R$ in $\%$.

\begin{tabular}{|c|c|c|c|c|c|c|c|c|c|c|c|}
\hline & (a) & (b) & s.u. & (c) & s.u. & (d) & s.u. & (e) & s.u. & (f) & s.u. \\
\hline noise* & off & off & & on & & on & & on & & on & \\
\hline 3rd order GC & P/Al & off & & $\mathrm{P} / \mathrm{Al}$ & & $\mathrm{P}$ & & $\mathrm{P}$ & & off & \\
\hline 4 th order GC & $\mathrm{P}$ & off & & $\mathrm{P}$ & & $\mathrm{P}$ & & off & & off & \\
\hline $\mathrm{d}(\mathrm{P}-\mathrm{C} 1)$ & 1.791 & 1.791 & & 1.791 & & 1.791 & & 1.791 & & 1.791 & \\
\hline $\mathrm{d}(\mathrm{P}-\mathrm{C} 6)$ & 1.786 & 1.786 & & 1.786 & & 1.786 & & 1.786 & & 1.786 & \\
\hline $\mathrm{x}$ & 0.270116 & 0.270027 & 0.000001 & 0.270100 & 0.000015 & 0.270101 & 0.000015 & 0.270102 & 0.000015 & 0.270023 & 0.000007 \\
\hline $\mathrm{y}$ & 0.704204 & 0.704138 & 0.000001 & 0.704204 & 0.000016 & 0.704206 & 0.000016 & 0.704208 & 0.000016 & 0.704132 & 0.000007 \\
\hline $\mathrm{z}$ & 0.401967 & 0.401986 & 0.000001 & 0.401974 & 0.000010 & 0.401974 & 0.000010 & 0.401975 & 0.000010 & 0.401987 & 0.000004 \\
\hline U11 & 0.022306 & 0.022018 & 0.000006 & 0.022202 & 0.000077 & 0.022201 & 0.000077 & 0.022039 & 0.000029 & 0.022035 & 0.000029 \\
\hline $\mathrm{U} 22$ & 0.017633 & 0.017668 & 0.000005 & 0.017465 & 0.000063 & 0.017467 & 0.000063 & 0.017660 & 0.000023 & 0.017655 & 0.000023 \\
\hline U33 & 0.016227 & 0.015992 & 0.000004 & 0.016080 & 0.000061 & 0.016080 & 0.000061 & 0.015964 & 0.000022 & 0.015960 & 0.000022 \\
\hline U12 & 0.002181 & 0.002081 & 0.000003 & 0.002197 & 0.000045 & 0.002197 & 0.000045 & 0.002090 & 0.000017 & 0.002089 & 0.000017 \\
\hline U13 & 0.000556 & 0.000599 & 0.000003 & 0.000528 & 0.000047 & 0.000528 & 0.000047 & 0.000610 & 0.000017 & 0.000612 & 0.000017 \\
\hline $\mathrm{U} 23$ & 0.002778 & 0.002660 & 0.000003 & 0.002803 & 0.000042 & 0.002803 & 0.000042 & 0.002649 & 0.000016 & 0.002649 & 0.000016 \\
\hline$U_{e q}$ & 0.019525 & 0.019342 & & 0.019385 & & 0.019385 & & 0.019335 & & 0.019330 & \\
\hline C111 & 0.000029 & - & - & 0.000031 & 0.000016 & 0.000033 & 0.000016 & 0.000033 & 0.000016 & - & - \\
\hline $\mathrm{C} 222$ & -0.000003 & - & - & 0.000011 & 0.000020 & 0.000012 & 0.000020 & 0.000015 & 0.000021 & - & - \\
\hline C333 & 0.000012 & - & - & 0.000013 & 0.000005 & 0.000013 & 0.000005 & 0.000013 & 0.000005 & - & - \\
\hline $\mathrm{C} 112$ & 0.000043 & - & - & 0.000045 & 0.000009 & 0.000045 & 0.000009 & 0.000045 & 0.000009 & - & - \\
\hline $\mathrm{C} 122$ & 0.000004 & - & - & -0.000008 & 0.000010 & -0.000008 & 0.000010 & -0.000008 & 0.000010 & - & - \\
\hline C113 & -0.000033 & - & - & -0.000024 & 0.000006 & -0.000024 & 0.000006 & -0.000023 & 0.000006 & - & - \\
\hline C133 & 0.000039 & - & - & 0.000035 & 0.000004 & 0.000036 & 0.000004 & 0.000036 & 0.000004 & - & - \\
\hline $\mathrm{C} 223$ & 0.000000 & - & - & -0.000004 & 0.000007 & -0.000004 & 0.000007 & -0.000004 & 0.000007 & - & - \\
\hline $\mathrm{C} 233$ & 0.000022 & - & - & 0.000018 & 0.000004 & 0.000018 & 0.000004 & 0.000019 & 0.000004 & - & - \\
\hline $\mathrm{C} 123$ & -0.000020 & - & - & -0.000024 & 0.000004 & -0.000024 & 0.000004 & -0.000024 & 0.000004 & - & - \\
\hline D1111 & 0.000039 & - & - & 0.000033 & 0.000010 & 0.000033 & 0.000010 & - & - & - & - \\
\hline D2222 & -0.000005 & - & - & -0.000034 & 0.000014 & -0.000034 & 0.000014 & - & - & - & - \\
\hline D3333 & 0.000007 & - & - & 0.000004 & 0.000002 & 0.000004 & 0.000002 & - & - & - & - \\
\hline D1112 & 0.000008 & - & - & 0.000008 & 0.000005 & 0.000008 & 0.000005 & - & - & - & - \\
\hline D1222 & 0.000006 & - & - & 0.000005 & 0.000006 & 0.000005 & 0.000006 & - & - & - & - \\
\hline D1113 & -0.000003 & - & - & 0.000000 & 0.000003 & 0.000000 & 0.000003 & - & - & - & - \\
\hline D1333 & 0.000001 & - & - & -0.000002 & 0.000001 & -0.000002 & 0.000001 & - & - & - & - \\
\hline D2223 & 0.000009 & - & - & 0.000014 & 0.000004 & 0.000014 & 0.000004 & - & - & - & - \\
\hline D2333 & 0.000005 & - & - & 0.000005 & 0.000002 & 0.000005 & 0.000002 & - & - & - & - \\
\hline D1122 & -0.000005 & - & - & -0.000013 & 0.000004 & -0.000013 & 0.000004 & - & - & - & - \\
\hline D1133 & 0.000006 & - & - & 0.000003 & 0.000002 & 0.000003 & 0.000002 & - & - & - & - \\
\hline D2233 & 0.000002 & - & - & 0.000000 & 0.000002 & 0.000000 & 0.000002 & - & - & - & - \\
\hline D1123 & 0.000001 & - & - & 0.000001 & 0.000002 & 0.000001 & 0.000002 & - & - & - & - \\
\hline D1223 & -0.000001 & - & - & -0.000005 & 0.000002 & -0.000005 & 0.000002 & - & - & - & - \\
\hline D1233 & 0.000003 & - & - & 0.000004 & 0.000001 & 0.000004 & 0.000001 & - & - & - & - \\
\hline
\end{tabular}




\begin{tabular}{|c|c|c|c|c|c|c|c|c|c|c|c|}
\hline & (a) & (b) & s.u. & (c) & s.u. & (d) & s.u. & (e) & s.u. & (f) & $\overline{\text { s.u. }}$. \\
\hline noise* & off & off & & on & & on & & on & & on & \\
\hline 3rd order GC & $\mathrm{P} / \mathrm{Al}$ & off & & $\mathrm{P} / \mathrm{Al}$ & & $\mathrm{P}$ & & $\mathrm{P}$ & & off & \\
\hline 4 th order GC & $\mathrm{P}$ & off & & $\mathrm{P}$ & & $\mathrm{P}$ & & off & & off & \\
\hline M1 & 4.727487 & 4.676929 & 0.006796 & 4.766778 & 0.035572 & 4.763719 & 0.035655 & 4.765609 & 0.035331 & 4.734058 & 0.035354 \\
\hline D1+ & -0.083755 & -0.071903 & 0.002139 & -0.085138 & 0.011760 & -0.085132 & 0.011786 & -0.088792 & 0.011736 & -0.072019 & 0.011081 \\
\hline D1- & -0.081504 & -0.085901 & 0.002178 & -0.090616 & 0.011817 & -0.090336 & 0.011843 & -0.090206 & 0.011819 & -0.096089 & 0.011270 \\
\hline D0 & 0.001121 & -0.025834 & 0.002116 & -0.020546 & 0.011616 & -0.020239 & 0.011640 & -0.020483 & 0.011636 & -0.042872 & 0.010900 \\
\hline Q0 & 0.132725 & 0.151436 & 0.002374 & 0.122856 & 0.013037 & 0.123176 & 0.013066 & 0.139514 & 0.012190 & 0.145481 & 0.012271 \\
\hline Q1+ & -0.013929 & -0.017503 & 0.001954 & -0.022464 & 0.010662 & -0.021950 & 0.010687 & -0.025255 & 0.010008 & -0.025081 & 0.010090 \\
\hline Q1- & -0.001107 & -0.007310 & 0.002109 & -0.005104 & 0.011417 & -0.005518 & 0.011442 & -0.014648 & 0.010818 & -0.013675 & 0.010907 \\
\hline Q2+ & -0.110834 & -0.117114 & 0.002352 & -0.114259 & 0.012862 & -0.114010 & 0.012890 & -0.121069 & 0.012077 & -0.119978 & 0.012161 \\
\hline Q2- & 0.052472 & 0.040644 & 0.002006 & 0.053452 & 0.010966 & 0.053727 & 0.010991 & 0.034707 & 0.010284 & 0.037451 & 0.010362 \\
\hline $\mathrm{O} 0$ & 0.015320 & -0.002187 & 0.002082 & 0.017933 & 0.011339 & 0.017865 & 0.011364 & 0.016603 & 0.011362 & 0.003446 & 0.010779 \\
\hline $\mathrm{O} 1+$ & -0.031655 & -0.004174 & 0.001948 & -0.052669 & 0.010597 & -0.053089 & 0.010621 & -0.052267 & 0.010613 & -0.022465 & 0.010070 \\
\hline O1- & -0.058460 & -0.018273 & 0.001928 & -0.045543 & 0.010443 & -0.045506 & 0.010465 & -0.044595 & 0.010456 & -0.010672 & 0.009958 \\
\hline $\mathrm{O} 2+$ & -0.004995 & -0.003752 & 0.001983 & -0.007394 & 0.010723 & -0.007718 & 0.010748 & -0.007556 & 0.010754 & -0.002912 & 0.010269 \\
\hline $\mathrm{O} 2-$ & 0.005727 & -0.012539 & 0.001879 & -0.001641 & 0.010138 & -0.001348 & 0.010161 & -0.001940 & 0.010171 & -0.017927 & 0.009726 \\
\hline $\mathrm{O} 3+$ & 0.163272 & 0.146733 & 0.001985 & 0.173834 & 0.010848 & 0.173897 & 0.010874 & 0.174648 & 0.010868 & 0.160071 & 0.010264 \\
\hline O3- & -0.059161 & -0.051805 & 0.001939 & -0.068841 & 0.010513 & -0.068893 & 0.010537 & -0.070104 & 0.010544 & -0.057580 & 0.010028 \\
\hline $\mathrm{HO}$ & 0.051373 & 0.058592 & 0.002302 & 0.056404 & 0.012818 & 0.056107 & 0.012846 & 0.059068 & 0.011798 & 0.055525 & 0.011903 \\
\hline $\mathrm{H} 1+$ & -0.014073 & -0.027446 & 0.002007 & 0.014347 & 0.011175 & 0.013370 & 0.011198 & 0.000989 & 0.010291 & 0.001473 & 0.010382 \\
\hline H1- & 0.008756 & 0.004319 & 0.002088 & -0.007637 & 0.011541 & -0.007029 & 0.011565 & -0.005786 & 0.010707 & -0.007037 & 0.010803 \\
\hline $\mathrm{H} 2+$ & -0.013680 & -0.017000 & 0.002134 & -0.032721 & 0.011775 & -0.032660 & 0.011801 & -0.038848 & 0.010945 & -0.037983 & 0.011037 \\
\hline H2- & 0.004100 & 0.004808 & 0.001952 & -0.002287 & 0.010782 & -0.002568 & 0.010805 & -0.002779 & 0.010003 & -0.001916 & 0.010092 \\
\hline $\mathrm{H} 3+$ & -0.015033 & -0.011876 & 0.001933 & -0.032426 & 0.010669 & -0.032488 & 0.010691 & -0.024275 & 0.009909 & -0.022989 & 0.009997 \\
\hline H3- & 0.022475 & 0.017539 & 0.001999 & 0.014204 & 0.011022 & 0.014560 & 0.011047 & 0.007084 & 0.010250 & 0.005785 & 0.010341 \\
\hline H4+ & 0.070642 & 0.066727 & 0.001889 & 0.083409 & 0.010453 & 0.084095 & 0.010477 & 0.081817 & 0.009683 & 0.079208 & 0.009763 \\
\hline H4- & 0.057957 & 0.060036 & 0.001843 & 0.049479 & 0.010199 & 0.049387 & 0.010223 & 0.052118 & 0.009457 & 0.051089 & 0.009531 \\
\hline$\kappa(P)$ & 1.079831 & 1.087263 & 0.000473 & 1.081432 & 0.002607 & 1.081448 & 0.002619 & 1.081018 & 0.002372 & 1.082829 & 0.002407 \\
\hline$\kappa^{\prime}(P)$ & 0.938526 & 0.938526 & - & 0.938526 & - & 0.938526 & - & 0.938526 & - & 0.938526 & - \\
\hline$R$ & 0.00 & 0.35 & & 1.54 & & 1.54 & & 1.55 & & 1.56 & \\
\hline
\end{tabular}

${ }^{*} p_{1}=0.333$

\section{Supplementary material to the probability density function of the $P$ atom}

The total integrated negative probability is $-0.008 \%$ and the associated volume $1.36 \AA^{3}$ for a $51 \times 51 \times 51$ cube around $\mathrm{P}$ with edge lengths $1.6 \AA$ and $\mathrm{P}$ in the center.

\section{Supplementary material to the section "Quadrupoles, octupoles and hexadecapoles"}

Figs. 1 to 3 show the histograms for the higher multipoles. The largest change due to a neglect of anharmonic nuclear motion is for refinement $\mathbf{b}$ due to a change in the octupoles, which contributes roughly $50 \%$ of the density parameter changes to the total parameter distance from the reference set of parameter values as measured by the parameter $R$-factor given in Table 3 in the main text. When noise enters the data (c), which in reality is always the case, however, the most dominant contribution comes from the hexadecapoles and the error contribution of the octupoles is markedly reduced. A particular strong contribution from $\mathrm{O} 3+$ is given.

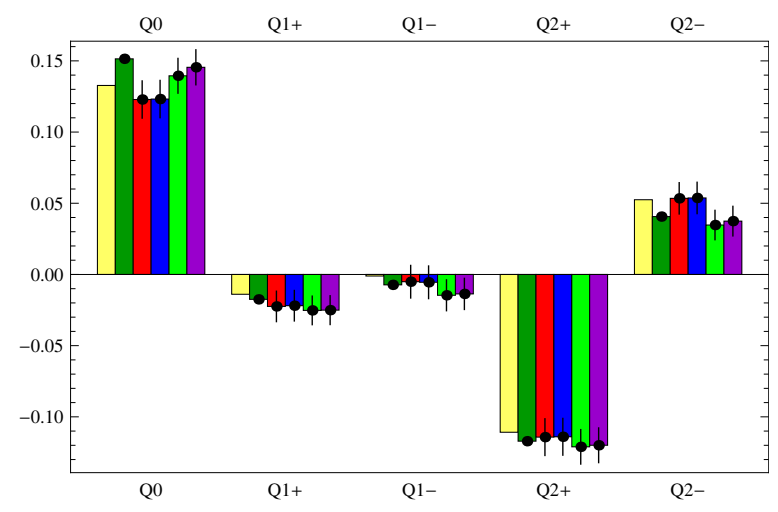

Figure 1

Quadrupole populations for refinements a-f. 


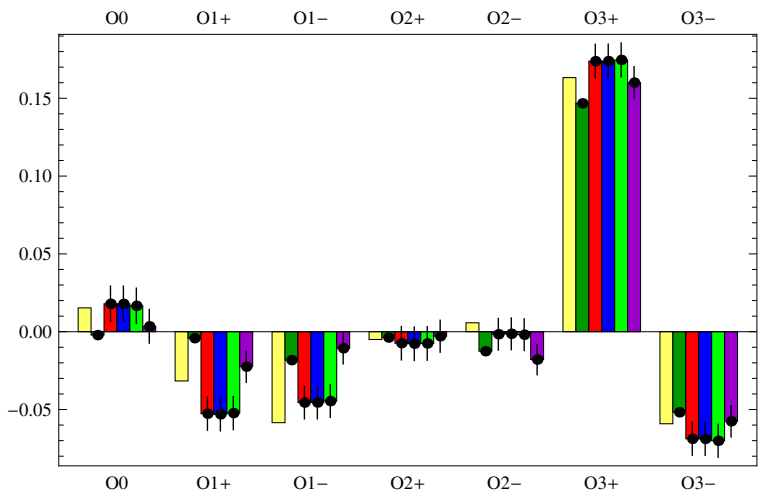

A pronounced effect on the octupoles can be observed, however, experimental noise induces larger changes in the hexadecapoles.

Figure 2

Octupole populations for refinements a-f.

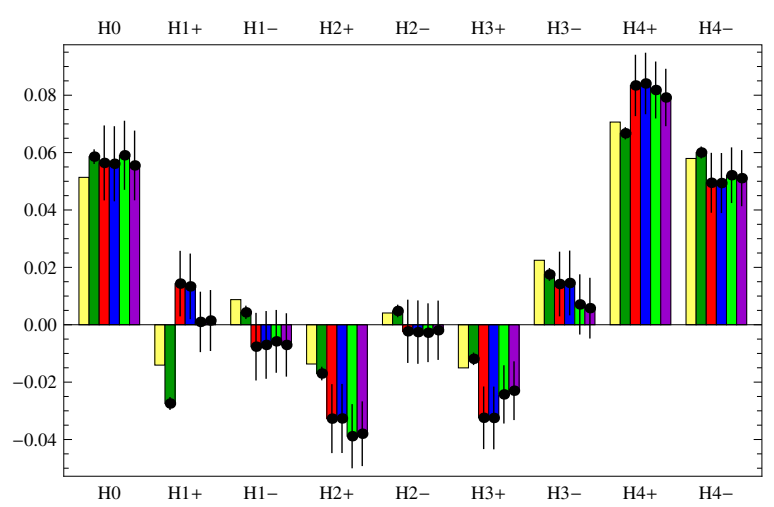

Figure 3

Hexadecapole populations for refinements a-f. 